\title{
Transient Characteristics of Water-Jet Propulsion with a Screw Mixed Pump during the Startup Process
}

\author{
Wei Han, ${ }^{1,2}$ Teng Zhang $\mathbb{D}^{1},{ }^{1}$ You Liang Su, ${ }^{1}$ Ran Chen, ${ }^{1}$ Yan Qiang, ${ }^{1}$ and Yang Han ${ }^{1}$ \\ ${ }^{1}$ Lanzhou University of Technology, College of Energy and Power Engineering, Lanzhou 730050, China \\ ${ }^{2}$ Key Laboratory of Fluid Machinery and Systems, Lanzhou 730050, China \\ Correspondence should be addressed to Teng Zhang; 18297669175@163.com
}

Received 16 March 2020; Accepted 15 April 2020; Published 4 May 2020

Academic Editor: Julien Bruchon

Copyright (c) 2020 Wei Han et al. This is an open access article distributed under the Creative Commons Attribution License, which permits unrestricted use, distribution, and reproduction in any medium, provided the original work is properly cited.

In order to investigate the transient hydrodynamic characteristics of water-jet propulsion with a screw mixed pump during the startup period and to overcome the difficulties in measuring the transient process parameters over a very short period of time, a method based on a flume experiment combined with computational fluid dynamics (CFD) is proposed. The thrust and torque of the pump-jet propulsion according to mooring status at different rotational speeds were measured by the test, which provided a group of data for the boundary and initial conditions of the numerical calculation of the user-defined function (UDF). Consecutive changes in the parameters of the water-jet propulsion dynamics can be captured from the numerical simulation of the startup process with the UDF. Thus, the transient hydrodynamic characteristics of water-jet propulsion according to time or rotation speed were obtained. The results show that the relationship between the thrust or torque of the water-jet propeller and pump rotational speed is close to the quadratic functions. The energy characteristic parameters of screw mixed water-jet pump, such as the flow rate, head, shaft power, and efficiency, rapidly increase and decrease and then remain relatively stable.

\section{Introduction}

As an advanced propulsion mode, thrust is generated by water-jet propulsion with a screw mixed pump relying mostly on the difference in momentum between the inlet and outlet water flows $[1,2]$. This method has many advantages over traditional propeller propulsion, such as higher efficiency, lower noise, more stable operation, and better resistance to cavitation, and it has been widely used in high-performance warships and new submarines. The thrust and power of waterjet propulsion with a screw mixed pump are usually altered by adjusting the rotational speed and are scarcely affected by the sailing speed. The rotational speed of the impeller rapidly reaches the designated value during the startup period, and the flow pattern changes from laminar to complex turbulent flow causing a rapid fluctuation in pressure, a high-water flow impact, and a transient high load on the blade which all affect the stability and longevity of the pump. Therefore, it is of great significance to investigate the transient characteristics of this type of water-jet propulsion during the startup period.
Zhang et al. [3] found that the quasi-steady calculations could not be used to accurately predict the transient behavior, especially in the initial stage of startup. Tsukamoto et al. $[4,5]$ found that the dimensionless head and flow rate began to decrease from initially large values, reaching steady-state values after a certain time below the steady-state curve during the centrifugal pump's startup processes. Chalghoum et al. [6] analyzed the effects of the impeller geometric parameters and different openings of the discharge valve on external characteristics during the centrifugal pump startup period. Li et al. [7] established a closed circulatory system with the centrifugal pump to study the transient characteristics during the startup period, which avoided the calculation error caused by the uncertainty regarding the boundary conditions of the inlet and outlet. Tanaka and Tsukamoto [8-10] studied cavitation in centrifugal pumps during the startup period by flow field visualization and found that the rapidly changing pressure and flow rate were related to the cavitation behavior of the pump. Based on the momentum moment theorem, Ping et al. [11] 
deduced the basic equation of steady speed and the starting process of a centrifugal pump, respectively, and described the influence of impeller rotating acceleration and fluid acceleration on the startup performance with a mathematical equation. Zhang et al. [12] qualitatively analyzed the transient behaviors in detail using the transient Euler equation of turbomachinery during all kinds of operation periods and quantitatively calculated the instantaneous additional theory heads and additional theory power. Wang et al. $[13,14]$ set up a model to analyze the startup process of the mixed-flow pump based on the internal characteristics of the pump and the one-dimensional unsteady motion equations of the pipeline. Li et al. [15] analyzed the starting characteristics of the mixed-flow pump based on a quasi-steady-state assumption, which provides a new idea for revealing the transient internal characteristics of the startup process of the mixed-flow pump. The transient behavior of the pump was analyzed using pump characteristic curves [16]. Li et al. [17] found that in the startup process of the mixed-flow pump, the flow stabilized in the impeller, the scale of vortex decreased gradually as the rotating speed approached the rated speed, and the transient effect disappeared.

The above-described investigation of water-jet propulsion pumps mainly focused on the characteristics and optimal design of the pump [18-20]. There is little research on the transient response characteristics of propulsion performance during the startup process for water-jet propulsion with a screw mixed pump. Fu et al. [21] found there are severe unsteady vortex flows in the vaneless space near the conditions under which the hydraulic torque on the runner equals to zero. Nouha et al. [22] found that the pump dynamic behavior with the impeller geometry during the starting periods. Tao et al. [23] found that the increase in the volute casing flow area is helpful to improve the operation stability of the pump. Wang et al. [24] found that the valve head change during the startup process had a significant transient effect. In this paper, the control of impeller speed realized by the userdefined function (UDF), in a numerical simulation, and a technique for controlling the frequency, in an experiment to reveal the transient hydrodynamic coefficients during the startup period of the water-jet pump, are described.

\section{Experiment for Pump-Jet Propulsion According to Mooring Status}

2.1. Experimental Equipment and Procedure. The experimental equipment mainly included digital torque and rotational speed measurers, a high-precision electronic force sensor, a model of a submarine pump, and a rotational speed governor, as shown in Figure 1. The components of the selfcirculating high-precision flume mainly included the flume body, the water supply equipment, the automatic flow measurement system, the tail door control system, and the ADV flow velocity measurement system. The length, width, and depth of the flume were $13,0.6$, and $0.6 \mathrm{~m}$, respectively. The flow rate of the flume was regulated by a closed-loop control mode, which has the characteristics of high accuracy and stable control and can meet the test requirements of steady and unsteady flows.

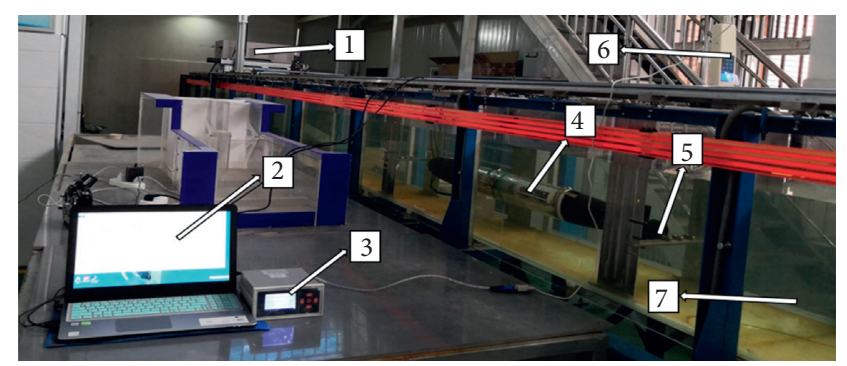

FIGURE 1: The experimental system of water-jet propulsion. (1) Ultrasonic velocimeter, (2) thrust collector, (3) speed torque indicator, (4) submersible model, (5) water-jet propulsion pump, (6) high-precision electronic force gauge, and (7) self-circulating experimental flume.

Firstly, the automatic flow measurement system and tail door control system were used to keep the water level of the flume at a fixed height. Secondly, the rotational speed of the water-jet pump was adjusted to a stable value by using the rotational speed governor. The rotational speed and corresponding torque were recorded using the digital torque and rotational speed measurers. The corresponding thrust value at the stable speed was recorded by the high-precision electronic force sensor and used. The arithmetic average value of the corresponding thrust was calculated by taking three measurements at the same rotational speed to reduce the error in the data.

2.2. Thrust and Torque at the Different Rotational Speeds. The experimental results show that the thrust and torque, at different rotational speeds, followed the quadratic function distribution, and the curves for thrust and torque at different rotational speeds are shown in Figure 2, and the mathematical relationship between thrust and torque is shown in equations (1) and (2):

$$
\begin{gathered}
T=-0.21859-8.43874 \times 10^{-4} n+4.50341 \times 10^{-6} n^{2}, \\
M=0.00926-4.24441 \times 10^{-5} n+1.57305 \times 10^{-7} n^{2} .
\end{gathered}
$$

\section{Numerical Simulation}

3.1. Geometric Model. The 3D geometric models and parameters of water-jet propulsion with a screw mixed pump are shown in Figure 3, which shows a 3-D model containing an impeller, a guide vane, and a nozzle. Table 1 lists the principal specifications of the water-jet pump.

3.2. Mesh Generation. Fully tetrahedral meshes were adapted to discretize the computational domains, as shown in Figure 4. The number of grids affects the accuracy of numerical calculations. In theory, the more the meshes there are, the higher the accuracy. Having too many meshes increases the calculation time and reduces the speed of convergence. Therefore, to eliminate the influence of the number of grids on the calculation of the results, it is necessary to check independence from the grid number. Six groups of time steps are selected for numerical calculation, as shown in Figure 5(a), as the time step increases, the head $h$ 


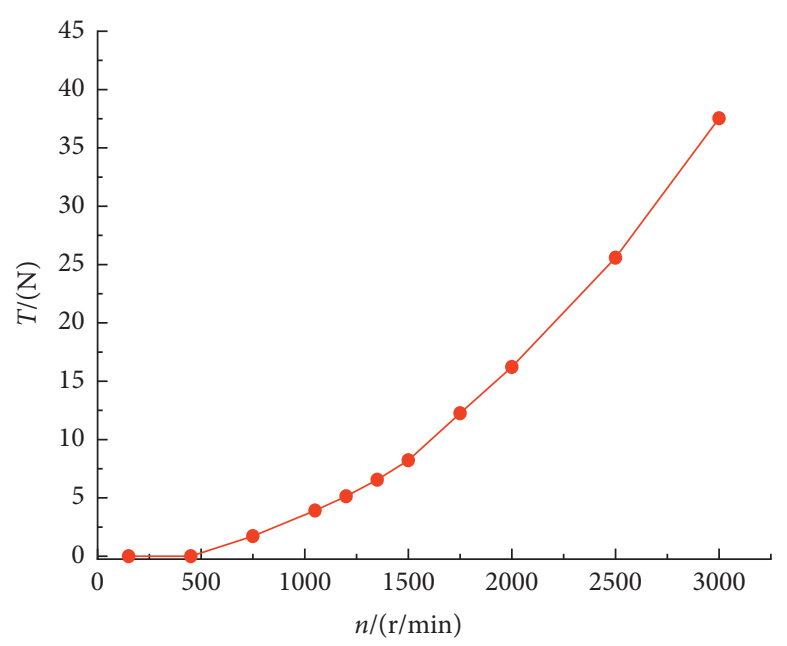

(a)

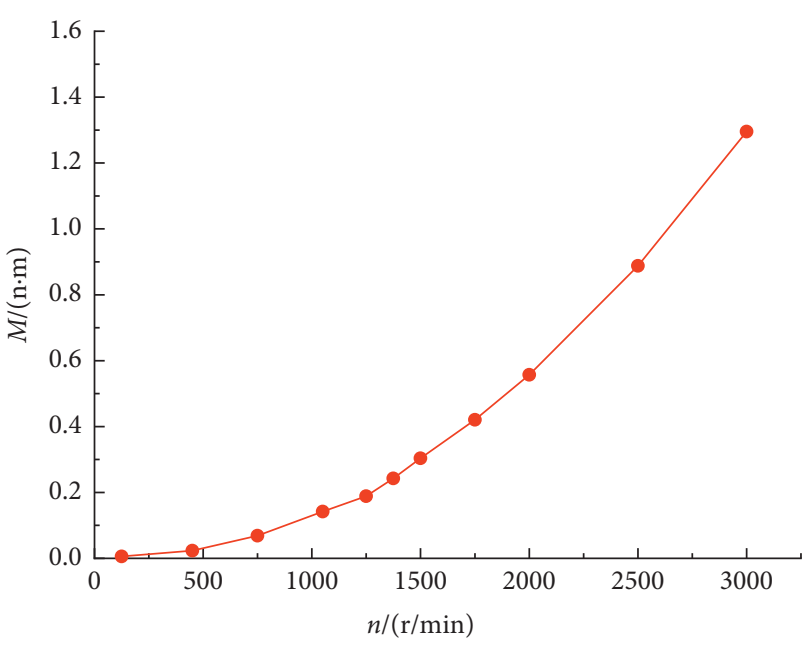

(b)

Figure 2: The variation of thrust and torque at the different rotational speeds: (a) thrust and (b) torque.

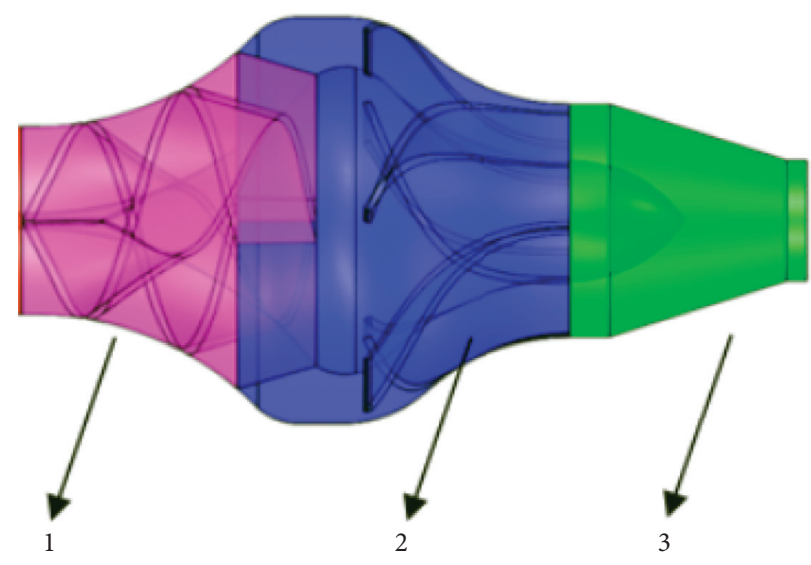

FIgURE 3: A 3-D model of water-jet propulsion with a screw mixed pump. (1) Impeller, (2) guide vane, and (3) nozzle.

TABLE 1: The design parameters of the water-jet pump model.

\begin{tabular}{lc}
\hline Geometric parameter & Value \\
\hline Flow rate & $13.8 \mathrm{~m}^{3} / \mathrm{h}$ \\
Head & $1.3 \mathrm{~m}$ \\
Rotational speed & $1500 \mathrm{rpm}$ \\
Thrust $(T / N)$ & 10.774 \\
Specific speed & 278 \\
\hline
\end{tabular}

gradually increases; the efficiency $\eta$ slowly increases with time, and when the mesh quantity is increased to 1.728 million, the head and efficiency of the water-jet pump remain stable.

\subsection{Verification of Independence from the Grid Number and} Time Step. To ensure the accuracy and precision of the numerical results, an appropriate time step is needed. In Figure 5(b), $T_{1}, T_{2}, T_{3}$, and $T_{4}$ are the numerical simulation results for thrust with time steps of $0.0008,0.0001,0.0002$, and $0.0004 \mathrm{~s}$, respectively, and $T_{5}$ is the experimental value when the system operation is stable. It can be seen from the figure that the errors for 0.00008 and 0.0004 s are $1.459 \%$ and $1.5125 \%$, respectively, while the errors for 0.0001 and $0.0002 \mathrm{~s}$ are smaller. To ensure the precision of the numerical calculations, $0.0001 \mathrm{~s}$ is selected as the time step.

\subsection{Numerical Model and Boundary Condition}

3.4.1. Governing Equations and Turbulence Model. In a reference-rotating frame related to the impeller passage, the basic equations of continuity and momentum are the following:

$$
\frac{\partial \rho}{\partial t}+\nabla\left(\rho v_{0}\right)=0
$$

$\frac{\mathrm{d}}{\mathrm{d} t}\left(\rho v_{0}\right)+2 \rho \omega \times v_{0}+\rho \omega \times \omega \times r=\rho F-\nabla p+\left(\mu+\mu_{t}\right) \nabla^{2} v_{0}$,

where $\rho, v_{0}, \omega, r, F, \mu, \mu_{t}$, and $\nabla$ are the density of the fluid, the velocity vector, the rotating angular velocity, the space vector, the mass force, the coefficient of kinetic viscosity, the coefficient of turbulent kinetic viscosity, and the Hamiltonian vector, respectively.

The RNG $\mathrm{k}-\varepsilon$ model was chosen as the turbulent model for numerical study. To realize the pressure speed coupling solution, two-order upwind schemes were adopted in the numerical calculation and the finite volume method was also used, as were the central difference schemes for the diffusion terms. The no-slip wall condition was applied for the walls of the hub and blade surface and for the other solid walls.

3.4.2. UDF and Boundary Conditions. The thrust and torque value at the stable speed following its change are obtained. The law of the changes in thrust and torque with the change in rotational speed is analyzed, and the determined rotational speed control law is provided for in the numerical simulation. The speed of the impeller was controlled in the process of numerical simulation. After the law of the integral 


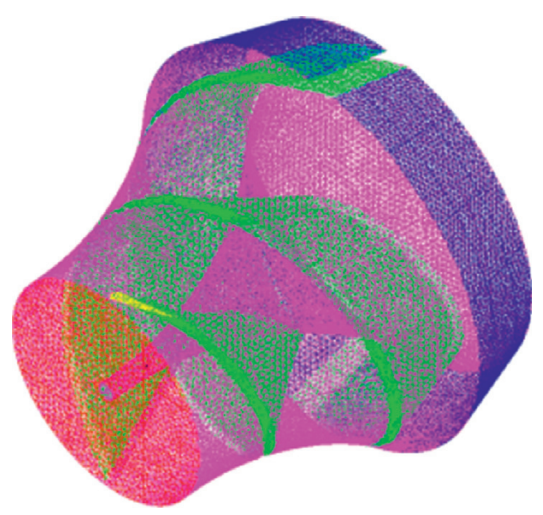

(a)

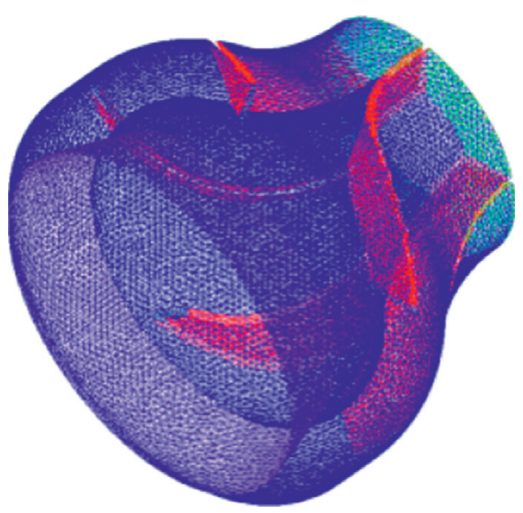

(b)

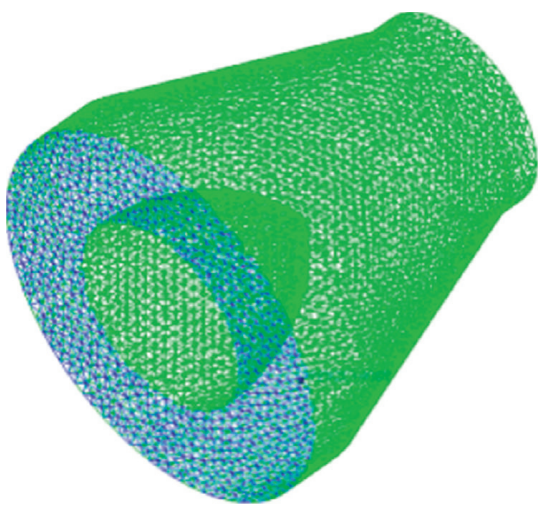

(c)

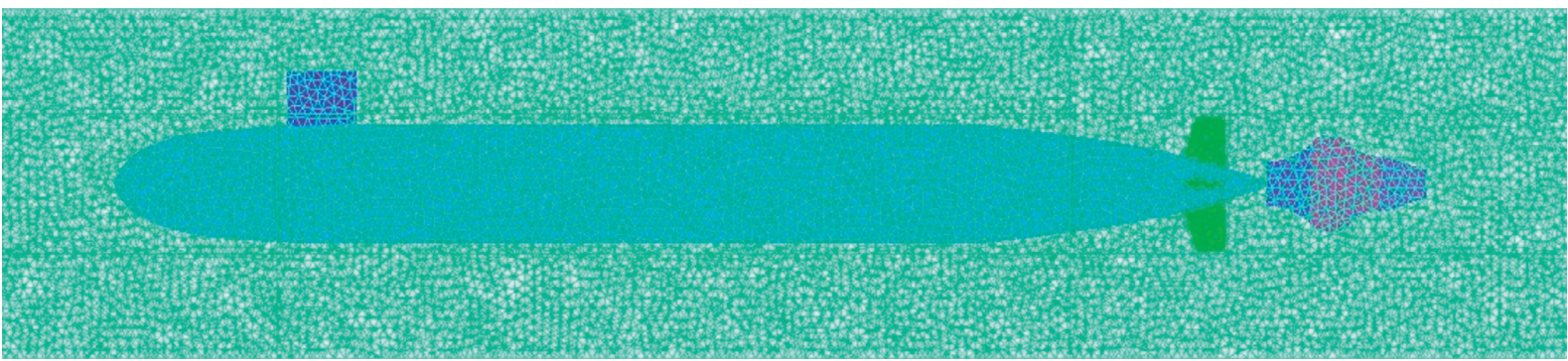

(d)

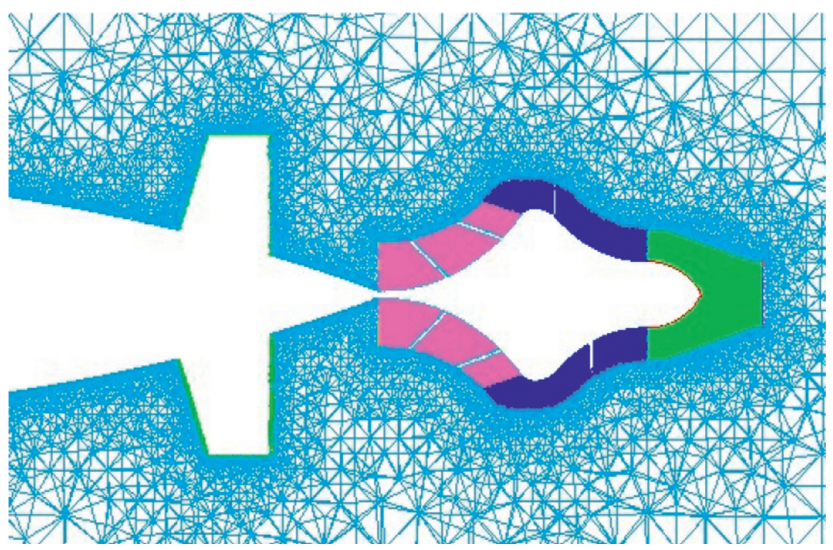

(e)

FIgURE 4: Computational domain meshes. (a) Impeller; (b) guide vane; (c) nozzle; (d) computing domain; (e) locally encryption mesh.

acceleration of the impeller was made discrete, a UDF program to control the change in speed was written. The program was loaded onto the impeller slip grid speed controller, and the steady-state result was taken as the initial condition of the unsteady calculation. In each iteration step of the unsteady calculation process, the impeller speed was updated. According to experimental statistics and related references [25], the variation of the rotational speed $n$ of the impeller can be described by equation (5); the change of $n$ with time $t$ is shown in Figure 6. To accurately capture the transient characteristics of the water-jet pump startup process, the computational time interval was set to $0.0001 \mathrm{~s}$. The maximum number of iterations for each time step was set to 20 and 7000 steps was calculated to ensure the stability of the internal flow:

$$
n= \begin{cases}15000 t, & t \leq 0.1 \mathrm{~s} \\ 1500, & t>0.1 \mathrm{~s}\end{cases}
$$

For the startup simulation, the contact surfaces between two adjacent components were connected through an interface boundary. The acceleration of the impeller was simulated using a sliding mesh method with rotational speeds assigned by a prescribed curve. In this study, the rotational speed increases linearly from zero to its rated speed of $1500 \mathrm{r} / \mathrm{min}$ in 0.1 seconds (marked as t0). After $0.1 \mathrm{~s}$, the speed of the impeller was constant. This was since the inlet and outlet boundary conditions for an individual three-dimensional pump model were unsteady during the startup process and because it is difficult to accurately specify the unsteady boundary conditions. To eliminate the errors of the inlet and outlet unsteady 


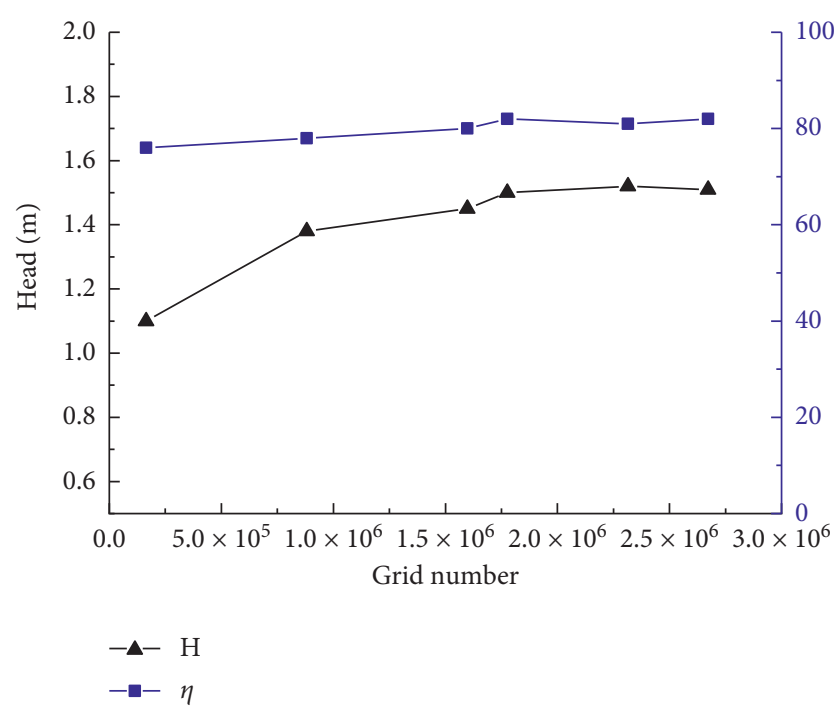

(a)

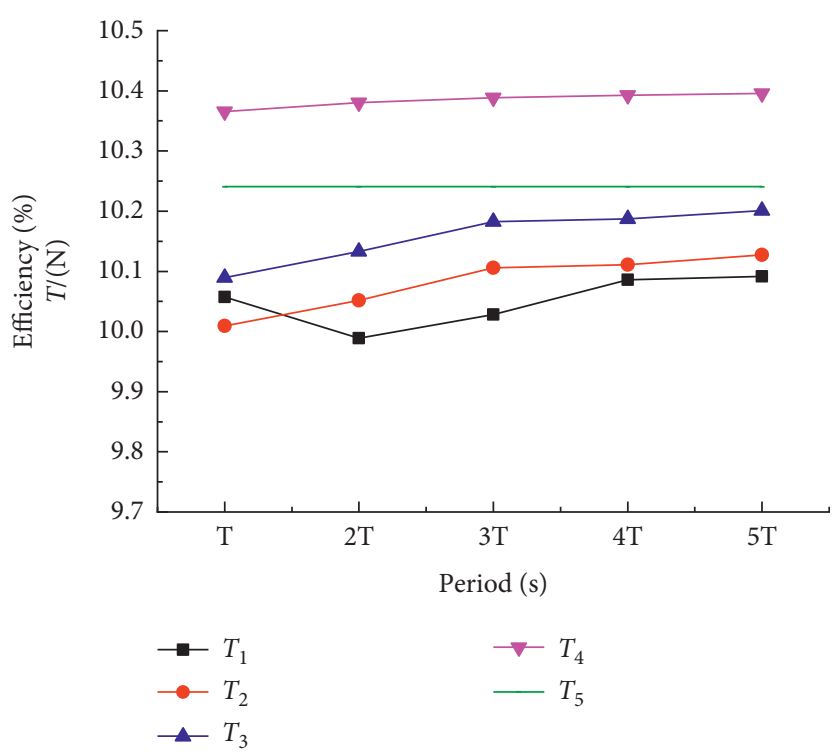

(b)

FIGURE 5: (a) Grid independence check; (b) time step independence check.

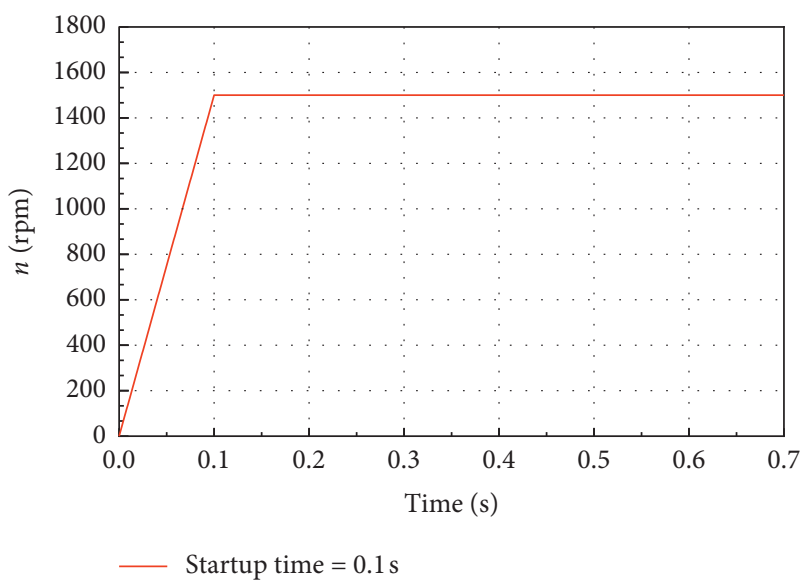

FIGURE 6: Impeller speed change curve of water-jet pump.

boundary conditions, a hypothesis was formulated, which assumed that the flow was stationary and that the pressure difference between the inlet and outlet was zero at the beginning of the startup. Based on the above assumption, the boundary conditions of the inlet and outlet were all set as total pressure stable and the reference pressure was set as 0 atm.

3.5. Comparison of Results from the Experiment and $\mathrm{Nu}$ merical Simulation. Figure 7 shows the 3-D model of the submarine pump; the computational domain size is $2000 \times 400 \times 400 \mathrm{~mm}$. Given the complexity of the submarine geometry, an unstructured tetrahedral mesh was used. The inlet of the computational domain extends, by three times the impeller outer diameter forward of the bow; the outlet extends, by four times the impeller outer diameter, to the rear of the submarine. The height and width of the computational domain are the same as those of the flume. For the numerical simulation of the whole fluid

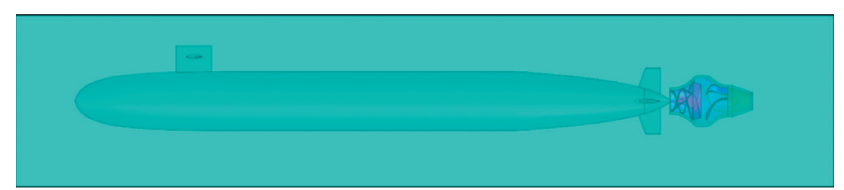

FIgURE 7: The 3-D model of the pump-jet submarine.

computational domain according to mooring status, the inlet was set as velocity and the outlet was set as the outflow.

The numerical simulation results for thrust and torque correspond to the average values of five cycles at each speed, and their relationships with pump speed are close to the quadratic functions. The comparison between the numerical simulation and experimental values of thrust and torque at variable rotational speeds is shown in Figure 8. As can be seen from Figure 8(a), the numerical simulation thrust values for water-jet propulsion with a screw mixed pump at different rotational speeds were larger than the corresponding experimental values. The total trend was consistent, and the error was limited to $3.2 \%$. This was mainly due to the existence of friction-based resistance from the experiment stand, the inability of water in the flume to remain absolutely stationary when measuring the thrust of the water-jet pump, and the fact that a part of the thrust was used to balance the drag of submarine. The torque of numerical simulation and the experimental values of the water-jet pump at various rotational speeds were compared in Figure 8(b). The maximum error between the numerical and experimental values of torque was $5.5 \%$, and the average error was $4.9 \%$. The numerical simulation was in good agreement with the data from the experiment, thus verifying the reliability of the numerical method.

\section{Results and Analysis}

4.1. Transient External Characteristic of the Water-Jet Propulsion Pump. During the startup stage, the inlet pressure $P_{1}$ 


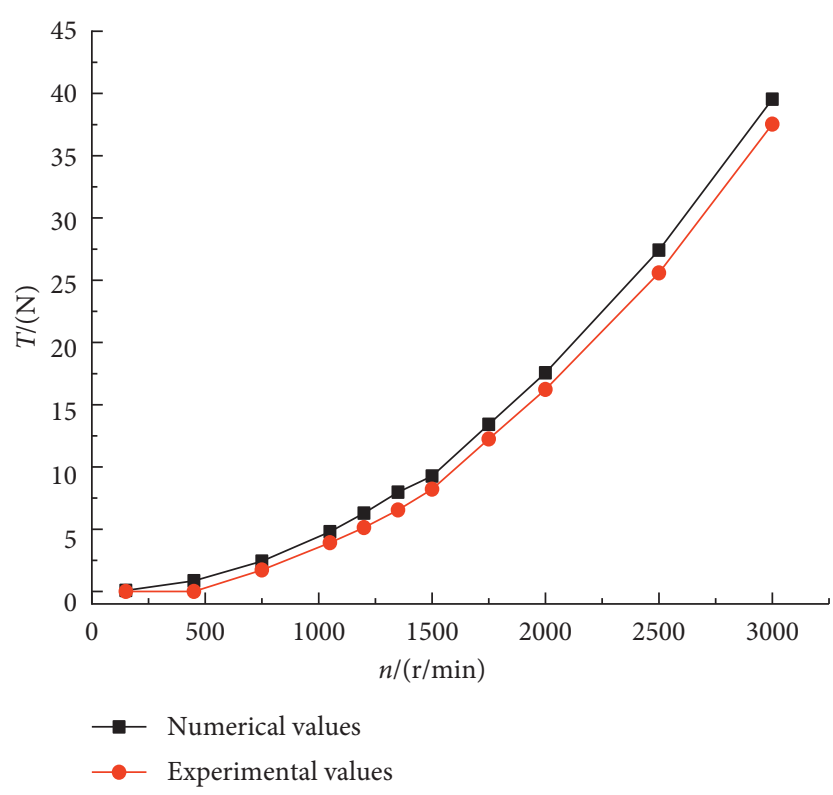

(a)

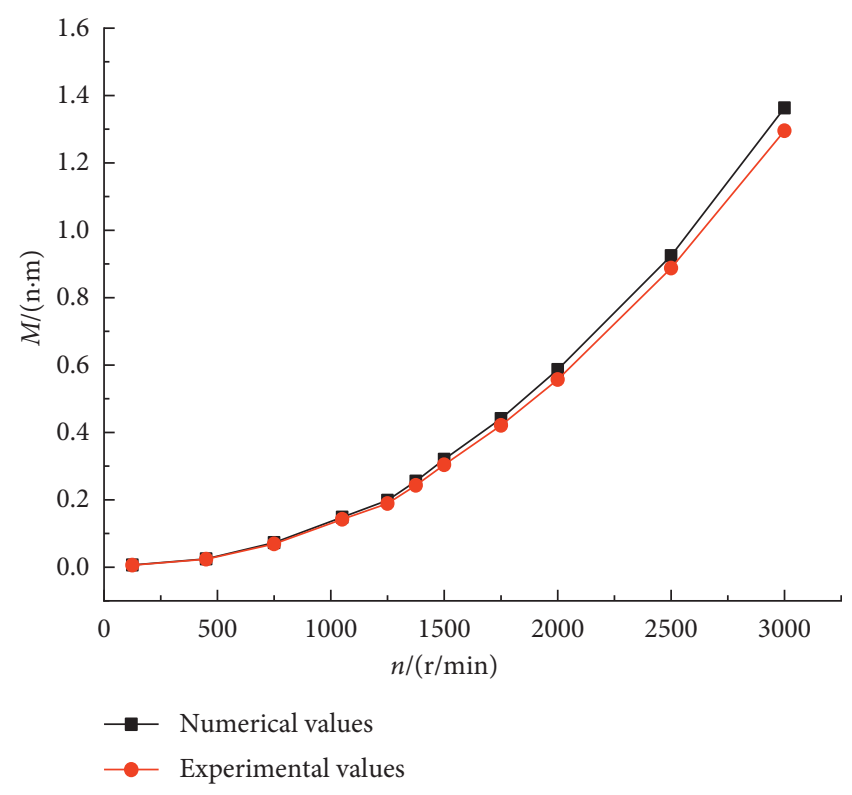

(b)

Figure 8: The comparison between the numerical and experimental values. (a) The $n$-T contrast curve of a submarine pump; (b) the $n$ - $M$ contrast curve of a submarine pump.

of the pump initially decreases and then rises to its peak, before gradually stabilizing. The outlet pressure $P_{2}$ rises rapidly to its peak value and then drops to a steady value, as shown in Figure 9(a). The flow rate of the pump gradually rises and tends to be stable, as shown in Figure 9(b). Compared with the change of head, there is no peak value of flow in the process of rising during the startup. The head of the water-jet propulsion pump is mainly determined by the inlet and outlet pressures. When the pump rotation is accelerated to the rated speed at $0.1 \mathrm{~s}$, the flow rate of the water-jet propulsion pump is $78.1 \%$ of the stable value. The changes in shaft power and efficiency are shown in Figure 9(c). Compared with the change of efficiency, the maximum value of shaft power occurs before the pump rotation speed reaches the rated speed. The change in thrust is more similar to the output characteristics of the step signal, while the injectionvelocity ratio is already close to the steady-state value at the beginning, as shown in Figure 9(d).

4.2. Dimensionless Parameter Analysis of the Water-Jet Pump during Startup. In order to eliminate the influence of the rotational speed on the external characteristics of the pump, the dimensionless head $\psi$ and flow rate $\phi$ were investigated to describe the transient responses, as shown in equation (4):

$$
\begin{aligned}
& \psi=\frac{2 g H}{u_{2}^{2}}, \\
& \phi=\frac{Q}{\pi d_{2} b_{2} u_{2}},
\end{aligned}
$$

where $Q$ is the instantaneous flow of the water-jet propulsion pump $\left(\mathrm{m}^{3} / \mathrm{s}\right), d_{2}$ is the outer diameter of the impeller $(\mathrm{m}), b_{2}$ is the impeller outlet width $(\mathrm{m}), H$ is the instantaneous head of the water-jet propulsion pump (m), and $u_{2}$ is the circular velocity of the impeller outlet $(\mathrm{m} / \mathrm{s})$.

The dimensionless head initially decreases and then rises to its peak value in the startup stage, as shown in Figure 10. As the rotational speed increases, the dimensionless head fluctuates within a large range. Compared with the dimensionless head, the dimensionless flow rate does not initially decrease during the startup phase.

4.3. Transient Response of the Propulsion Dynamic Characteristics of the Pump during Startup. In order to analyze the propulsion dynamic characteristics of the water-jet propulsion pump during startup, the changes in the characteristic coefficients of water-jet propulsion with time were used to describe the startup performance. The coefficients are the inlet velocity coefficient $J$, thrust coefficient $K_{t}$, moment coefficient $K_{q}$, and open-water efficiency $\eta_{0}$, respectively, as shown in the following equation:

$$
\begin{gathered}
J=\frac{v_{1}}{n D_{d}}, \\
K_{t}=\frac{T}{\rho n^{2} D_{d}^{4}}, \\
K_{q}=\frac{M}{\rho n^{2} D_{d}^{5}}, \\
\eta_{0}=\frac{J}{2 \pi} \frac{K_{t}}{K_{q}},
\end{gathered}
$$

where $v_{1}, n, D_{d}, \rho, T$, and $M$ are the inlet velocity, rotation speed, external diameter, fluid density, thrust, and torque of the pump, respectively.

To obtain the transient response curve of the coefficients of the water-jet propulsion dynamic characteristics, the 


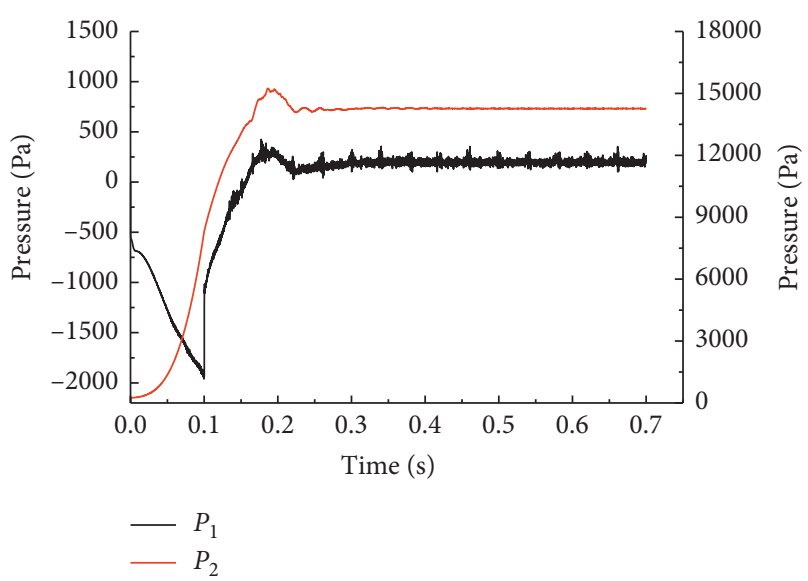

(a)

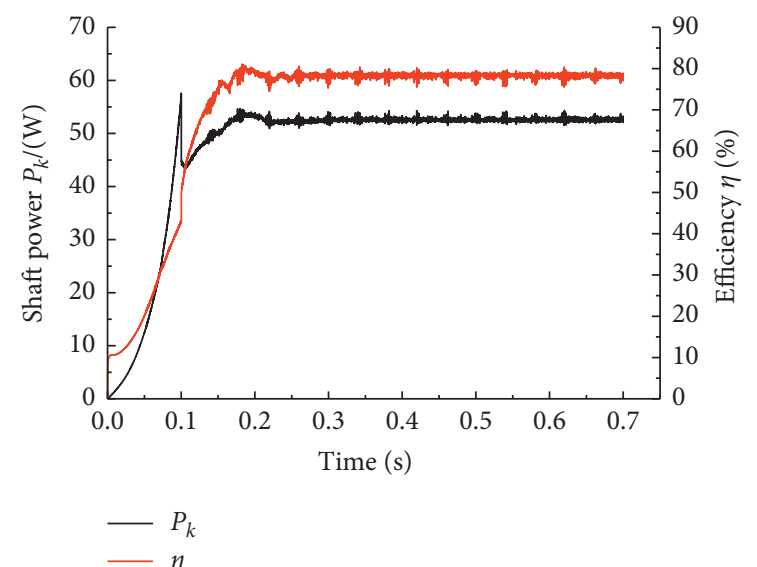

(c)

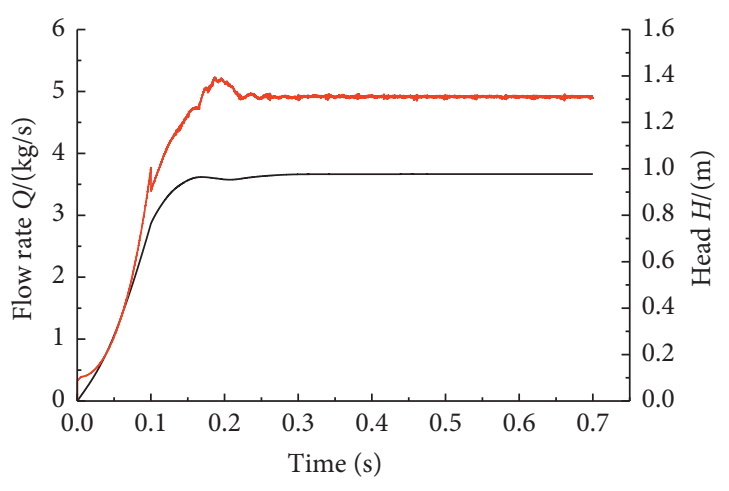

$-T$

$-K$

(b)

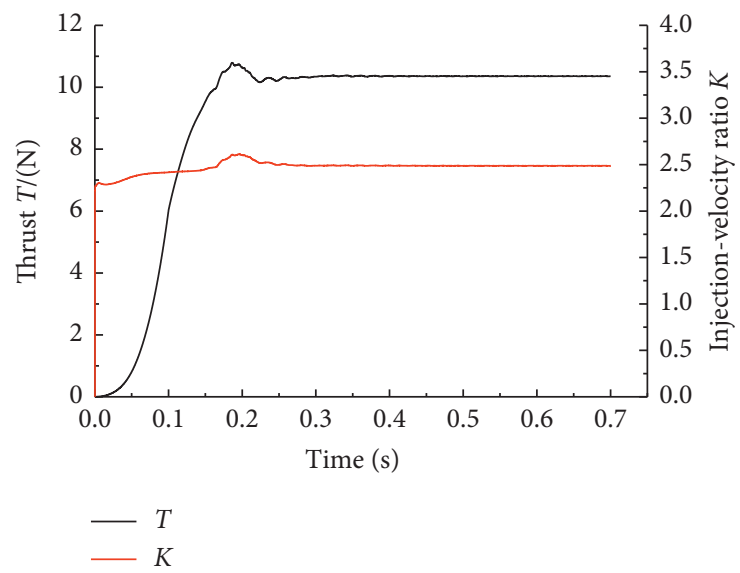

(d)

Figure 9: The transient external characteristic curve of the water-jet pump during the startup process. (a) $P$ curve, (b) $Q-H$ curve, (c) $P_{k^{-}} \eta$ curve, and (d) $T-K$ curve.

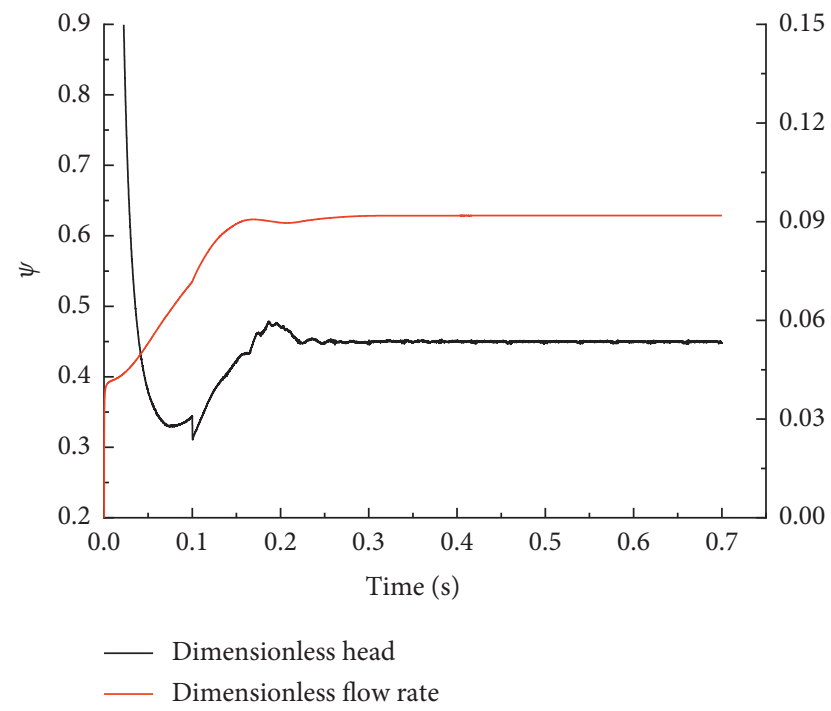

FiguRE 10: The dimensionless parameter variation curve of the water-jet propulsion pump. 


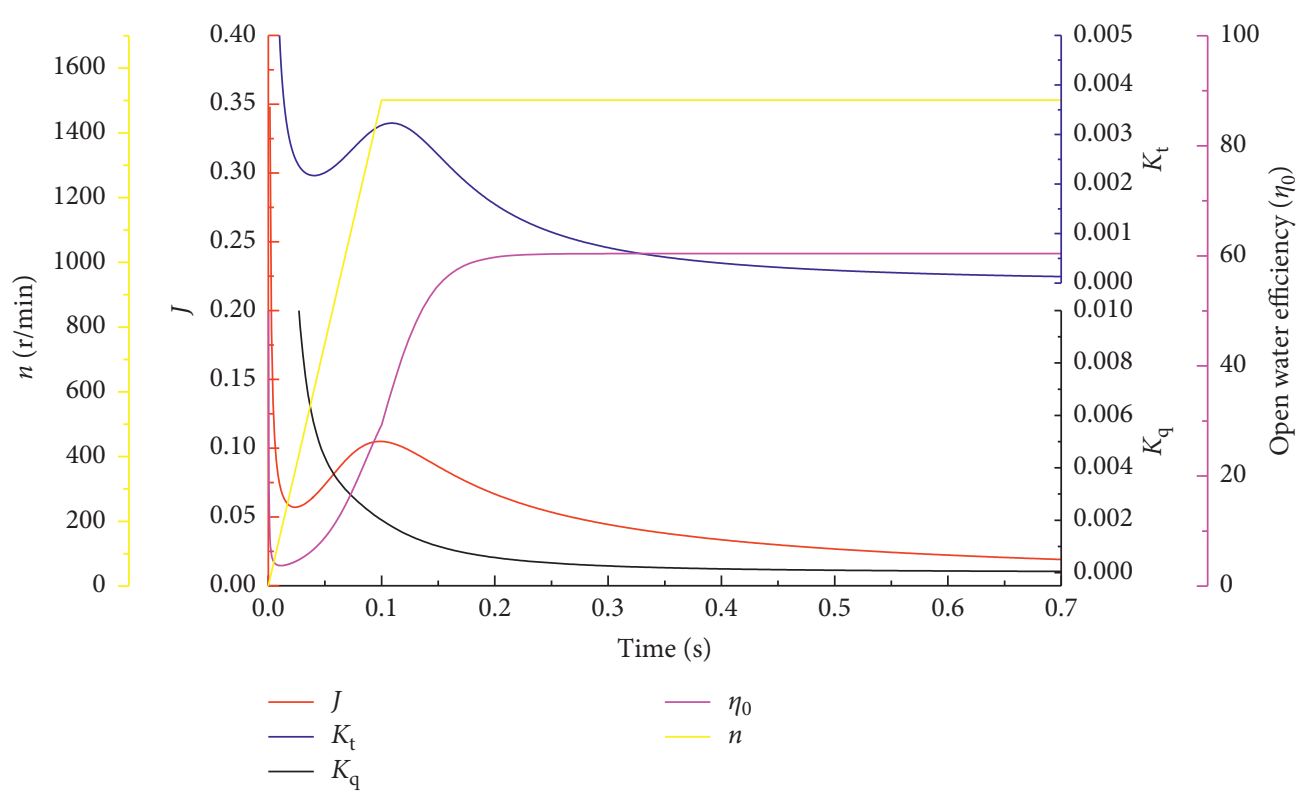

FIGURE 11: Transient response curves of the coefficients of the dynamic characteristics of water-jet propulsion with a screw mixed pump during the startup period.

variations of the inlet velocity, thrust, and torque with time can be fitted, as shown in Figures 6 and 9. The expression of the transient propulsion characteristic parameters can be described as follows:

$$
\left\{\begin{array}{l}
v_{1}(t)=\frac{1.9}{1+e^{3.2-45 t}}-0.01 \\
T(t)=\frac{10.5}{1+e^{4.4-45 t}}-0.1 \\
M(t)=\frac{0.33}{1.2+e^{3-45 t}}+0.06
\end{array}\right.
$$

The expressions of the transient propulsion characteristic parameters with impeller rotation speed can be deduced from equations (8) coupling with equation (5), as follows:

$$
\left\{\begin{array}{l}
v_{1}(n)=\frac{1.9}{1+e^{3.2-(3 / 1000) n}}-0.01 \\
T(n)=\frac{10.5}{1+e^{4.4-(3 / 1000) n}}-0.1 \\
M(n)=\frac{0.33}{1.2+e^{3-(3 / 1000) n}}+0.06
\end{array}\right.
$$

By substituting equations (8) into equation (7), the transient response of the water-jet propulsion dynamic characteristics during startup can be obtained, as shown in Figure 11.

As the motor reaches its stable speed, the inlet velocity coefficient and the thrust coefficient decrease rapidly from the maximum value and then increase slowly, shown in Figure 10. After the impeller speed stabilizes, they begin to decrease. The water-jet propulsion with a screw mixed pump enters its stable operation stage. Compared with the rotational speed, the inlet velocity and thrust coefficients lag behind in the startup phase. After the impeller rotation speed has stabilized, the response curve of the moment coefficient gradually decreases and has no oscillation process. However, the open-water efficiency plunges to its minimum value at the initial time and then gradually increases to a stable value.

\section{Conclusions}

The transient characteristics of the screw mixed-flow waterjet propulsion pump during its startup were revealed in this paper by a method coupling experiments and numerical simulation. The main conclusions are as follows:

(1) A propulsion dynamic model and modeling methods for the transient response of water-jet propulsion with a screw mixed pump during the startup period were proposed based on the parameters transformation.

(2) During the startup process, the transient external characteristics of the water-jet pump, including the flow rate, head, and shaft power, are analogous to the step inputs for the transient response characterized by time, which have obvious transient characteristics, including a rise time, a peak time, a delay time, and oscillation times.

(3) The slope and step responses of the thrust and torque to changes in the rotational speed can be described by a set of exponential functions.

(4) Before the motor speed is stable, the values of the water-jet propulsion dynamic parameters drop rapidly and then gradually stabilize. The inlet speed and thrust coefficients reach their peak values after the 
motor speed stabilizes. Otherwise, the moment coefficient and open-water efficiency have no oscillation process after the motor reaches a stable speed.

\section{Data Availability}

The data used to support the findings of this study are included within the article. Results of this paper can be verified according to the data and methods given in the paper.

\section{Conflicts of Interest}

The authors declare that they have no conflicts of interest.

\section{Authors' Contributions}

W. H. conceived and designed the research, T. Z. and Y. H. drafted the article, Y. S. completed the English check, R. C. processed the data, and Y. Q. performed the literature search.

\section{Acknowledgments}

This study was supported by the National Natural Science Foundation of China (grant nos. 51669012 and 51966010) and the National Key R\&D Program Projects of China (grant no. 2018YFB0606100).

\section{References}

[1] Y. Y. Ni and W. M. Liu, "Overview on research of water-jet propulsion," Ship \& Ocean Engineering, vol. 42, pp. 1-5, 2013.

[2] L. L. Cao, B. X. Che, and L. J. Hu, "Design method of water jet pump towards high cavitation performances," IOP Conference Series: Materials Science and Engineering, vol. 129, Article ID 012067, 2016.

[3] Y. L. Zhang, Z. C. Zhu, H. S. Dou et al., "Numerical investigation of transient flow in a prototype centrifugal pump during startup period," International Journal of Turbo \& JetEngines, vol. 34, no. 2, pp. 167-176, 2016.

[4] H. Tsukamoto and H. Ohashi, "Transient characteristics of a centrifugal pump during starting period," Journal of Fluids Engineering, vol. 104, no. 1, pp. 6-13, 1982.

[5] H. Tsukamoto, S. Matsunaga, H. Yoneda, and S. Hata, "Transient characteristics of a centrifugal pump during stopping period," Journal of Fluids Engineering, vol. 108, no. 4, pp. 392-399, 1986.

[6] I. Chalghoum, S. Elaoud, M. Akrout, and E. H. Taieb, "Transient behavior of a centrifugal pump during starting period," Applied Acoustics, vol. 109, pp. 82-89, 2016.

[7] Z. Li, D. Wu, and L. Wang, "Numerical simulation of the transient flow in a centrifugal pump during starting period," Journal of Fluids Engineering, vol. 132, Article ID 081102, 2010.

[8] T. Tanaka and H. Tsukamoto, "Transient behavior of a cavitating centrifugal pump at rapid change in operating conditions-part 1: transient phenomena at opening/closure of discharge valve," Journal of Fluids Engineering, vol. 121, no. 4, pp. 841-849, 1999.

[9] T. Tanaka and H. Tsukamoto, "Transient behavior of a cavitating centrifugal pump at rapid change in operating conditions-part 2: transient phenomena at pump startup/ shutdown," Journal of Fluids Engineering, vol. 121, no. 4, pp. 850-856, 1999.
[10] T. Tanaka and H. Tsukamoto, "Transient behavior of a cavitating centrifugal pump at rapid change in operating conditions-part 3: classifications of transient phenomena," Journal of Fluids Engineering, vol. 121, no. 4, pp. 857-865, 1999.

[11] S. L. Ping, D. Z. Wu, and L. Q. Wu, "Transient effect analysis of centrifugal pump during rapid starting period," Journal of Zhejiang University, vol. 41, pp. 814-817, 2007.

[12] Y. L. Zhang, Z. C. Zhu, and H. C. Lin, "The calculation of additional theory head generated by centrifugal pump during startup period," Chinese Quarterly of Mechanics, vol. 33, pp. 456-460, 2012.

[13] L. Q. Wang, D. Z. Wu, S. Y. Zheng et al., "Test and numerical calculation of transient performance of mixed-flow-pump during starting period," Journal of Zhejiang University, vol. 38, pp. 100-104, 2004.

[14] L. Q. Wang, D. Z. Wu, and S. Y. Zheng, "Experimental study on transient performance of a mixed-flow-pump," Journal of Fluid Mechanics, vol. 31, pp. 1-3, 2003.

[15] W. Li, L. L. Ji, and W. D. Shi, "Starting characteristic of mixedflow pump based on quasi-steady state assumption," Transactions of the Chinese Society of Agricultural Engineering, vol. 32, no. 7, pp. 86-92, 2016.

[16] K. Farhadi, "Transient behaviour of a parallel pump in nuclear research reactors," Progress in Nuclear Energy, vol. 53, no. 2, pp. 195-199, 2011.

[17] W. Li, Y. Zhang, W. Shi, L. Ji, Y. Yang, and Y. Ping, "Numerical simulation of transient flow field in a mixed-flow pump during starting period," International Journal of $\mathrm{Nu}$ merical Methods for Heat and Fluid Flow, vol. 28, no. 4, 2018.

[18] J. A. Han, C. Li, and J. J. Zhong, "Hydraulic design and performance analysis of waterjet axial flow pump," Journal of Engineering Thermophysics, vol. 35, pp. 2412-2416, 2014.

[19] D. Huang, Z. Y. Pan, and X. W. Pan, "Numerical simulation of cavitation flow in counter-rotating axial-flow pump," Journal of Drainage and Irrigation Machinery Engineering, vol. 33, no. 4, pp. 311-315, 2015.

[20] Y. Wang, P. Y. Cao, and G. Yin, "Flow feature and blade loading of a water-jet pump under non-uniform suction flow," Journal of Propulsion Technology, vol. 38, pp. 75-81, 2017.

[21] X. Fu, D. Li, H. Wang, G. Zhang, Z. Li, and X. Wei, “Analysis of transient flow in a pump-turbine during the load rejection process," Journal of Mechanical Science and Technology, vol. 32, no. 5, pp. 2069-2078, 2018.

[22] K. Nouha, E. Sami, C. Issa et al., "Effects of starting time and impeller geometry on the hydraulic performance of a centrifugal pump," in Proceedings of the International Conference Design \& Modeling of Mechanical Systems, Springer, Cham, Switzerland, 2018.

[23] Y. Tao, S. Yuan, J. Liu et al., "Influence of cross-sectional flow area of annular volute casing on transient characteristics of ceramic centrifugal pump," Chinese Journal of Mechanical Engineering, vol. 32, no. 1, 2019.

[24] Y. Wang, J. Chen, H. Liu et al., "Transient characteristic analysis of ultra-low specific-speed centrifugal pumps during startup period under shut-off condition," Transactions of the Chinese Society of Agricultural Engineering, vol. 33, no. 11, pp. 68-74, 2017.

[25] Z. Li, P. Wu, D. Wu, and L. Wang, "Experimental and numerical study of transient flow in a centrifugal pump during startup," Journal of Mechanical Science and Technology, vol. 25, no. 3, pp. 749-757, 2011. 